\title{
Investigating Human-Human Approach and Hand-Over
}

\author{
Patrizia Basili, Markus Huber, Thomas Brandt, Sandra Hirche, Stefan Glasauer
}

\begin{abstract}
Humans interact safely, effortlessly, and intuitively with each other. An efficient robot assistant should thus be able to interact in the same way. This requires not only that the robot can react appropriately to human behaviour, but also that robotic behaviour can be understood intuitively by the human partners. The latter can be achieved by the robot mimicking certain aspects of human behaviour so that the human partner can more easily infer the intentions of the robot. Here we investigate a simple interaction scenario, approach and hand-over, to gain better understanding of the behavioural patterns in human-human interactions. In our experiment, one human subject, holding an object, approached another subject with the goal to hand over the object. Head and object positions were measured with a motion tracking device to analyse the behaviour of the approaching human. Interaction indicated by lifting the object in order to prepare for hand-over started approximately $1.2 \mathrm{~s}$ before the actual hand-over. Interpersonal distance varied considerably between subjects with an average of $1.16 \mathrm{~m}$. To test whether the behavioural patterns observed depended on two humans being present, we replaced in a second experiment the receiving subject with a table. We found that the behaviour of the transferring subject was very similar in both scenarios. Thus, the presence of the receiving subject plays a minor role in determining parameters such as start of interaction or interaction distance. We aim to implement and test the parameters derived experimentally in a robotic assistant to improve and facilitate human-robot interaction.
\end{abstract}

Patrizia Basili, Markus Huber, Stefan Glasauer

Center for Sensorimotor Research, Institute of Clinical Neurosciences, Ludwig-MaximiliansUniversität München, e-mail: \{p.basili,markus.huber,S.Glasauer\}@lrz.uni-muenchen.de Thomas Brandt

Chair of Clinical Neurosciences, Ludwig-Maximilians-Universität München e-mail: thomas.brandt@med.uni-muenchen.de

Sandra Hirche

Institute of Automatic Control Engineering, Technische Universität München e-mail: hirche@1sr.ei.tum.de 


\section{Introduction}

According to the human-centered approach to robotics, efficient human-robot interaction can be achieved by the robot mimicking crucial aspects of human behaviour [5]. This enables the human partners to intuitively infer the intentions of the robot and thus to predict its further actions. An interaction between humans can be described by a set of algorithms which allow a natural, effective and safe interaction. For a robot, this set needs to be specified. The aim is therefore to define and to implement mathematical models derived from human-human experiments in autonomous robots. Here we aim to quantitatively describe one aspect of human interaction with the goal to implement the results in the robotic system presented in [2] (see figure 1). Until now, several studies were done on human-robot interaction [1],[3],[4], [9]-[15] but only few of them [3],[9],[10],[12] were based on the analysis of humanhuman experiments. Our previous investigations on a simple hand-over scenario [9] demonstrated that human-robot interaction can indeed be improved significantly by implementing simple modifications to the robot behaviour such as human-like motion profiles following a minimum-jerk trajectory. Here, we extend our investigation to the approach phase preceding the hand-over. The case of a robot approaching a person for interaction has been investigated previously by others ([4],[11],[13],[15]), but the main parameters for the interaction, such as interpersonal distance, were usually taken from results presented by the anthropologist T.E. Hall [6] in the 1960's. According to Hall, the distance between humans in an interaction depends on their emotional relationship and cultural background. In order to test these findings and to possibly define better human approach criteria, we investigated two experimental setups. In the first setup, one person, the transferring subject, walked towards a standing person, the receiving subject, to hand over an object. In the second setup, the receiving subject was replaced by a table.

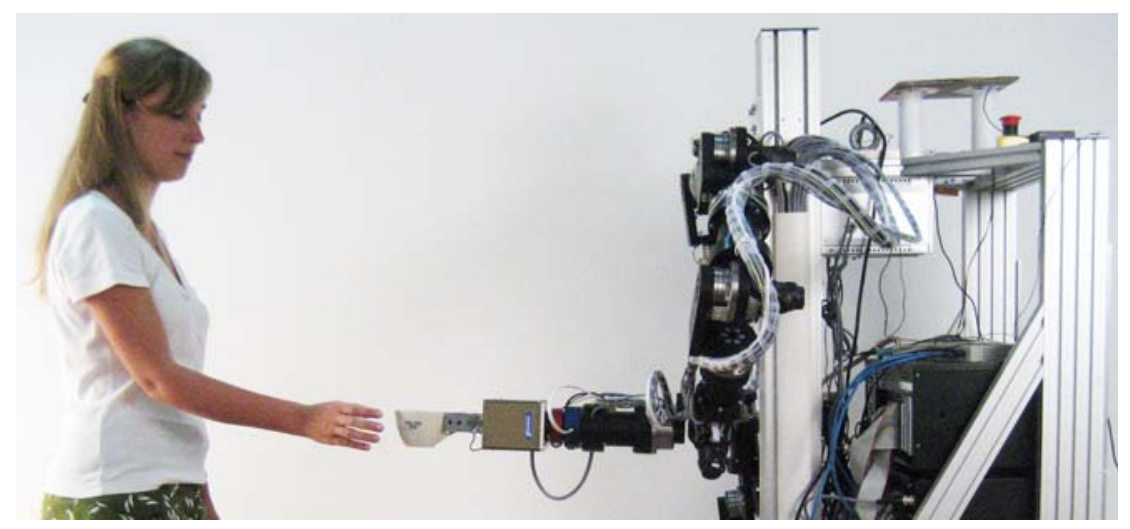

Fig. 1 Human-robot interaction scenario. 


\section{Methods}

\subsection{Experimental Setup}

We measured hand movements in human subjects during an approach and handover task using a motion tracking system (IS-600 Mark 2, InterSense Inc., USA). The size of the tracking area was $3 m \times 6 m$ in the middle of a room of $38 m^{2}$. We tested 26 pairs of subjects ( 6 female, 20 male, all right-handed). Each pair of subjects performed 4 consecutive trials. The item to be handed-over was a half full $0.5 \mathrm{~L}$ plastic bottle with a height of $17 \mathrm{~cm}$ and a diameter of $10 \mathrm{~cm}$ with a weight of 210 g. Each subject served once as a standing person (receiving subject, R), and once as an approaching person (transporting subject, $\mathrm{T}$ ). $\mathrm{R}$ and $\mathrm{T}$ were placed on predefined positions with a distance of 4.2 metres between them. $R$ was instructed to stand on her position aware that $\mathrm{T}$ will give her an object. $\mathrm{T}$ was told to walk from the specified starting position towards $\mathrm{R}$ in order to deliver the bottle. Neither the speed nor the approaching path towards $\mathrm{R}$ was specified in order not to affect the natural behavior of $\mathrm{T}$. The head position and orientation of the approaching person $\mathrm{T}$ was recorded by the tracking system. An additional tracking sensor, representing T's hand position, was placed on the item which was handed over. The head position of T was sampled with $150 \mathrm{~Hz}$, the hand/object position with $20-50 \mathrm{~Hz}$ due to technical limitations of the tracking system. The recording of the data during each trial started with $\mathrm{T}$ already holding the object in his hand and finished when both R's and T's hands were on the object.

In a second setup the standing person $\mathrm{R}$ was replaced by a table. Here, 24 subjects were tested (6 female and 18 male, all right-handed). In this second scenario, T had to place the object on the table, which had a height of $1.2-1.3 \mathrm{~m}$ that was individually derived from the first experiment in order to match the height of the hand-over in the first experiment. The table was placed in the same position as $\mathrm{R}$ in the first experiment. $\mathrm{T}$ received the instructions to go from the same starting position, as in the previous experiment, towards the table and to put the item on a specified position on the table. A tabletop of about $0.1 \times 0.1 \mathrm{~m}^{2}$ assured a well-defined position for placing the object (the same bottle as in experiment 1).

\subsection{Data Analysis}

The approach and hand-over interaction was analysed off-line by deriving several parameters from the measured raw data using Matlab (The Mathworks, Natick, MA). The time and interpersonal distance of the start of the interaction was defined as the start of the hand movement in subject-coincident co-ordinates from a combination of several criteria (see figure 2): the distance between R and T's hand had to start to decrease rapidly compared to the interpersonal distance, the hand 
speed of T increased, and the height above ground of T's hand increased.

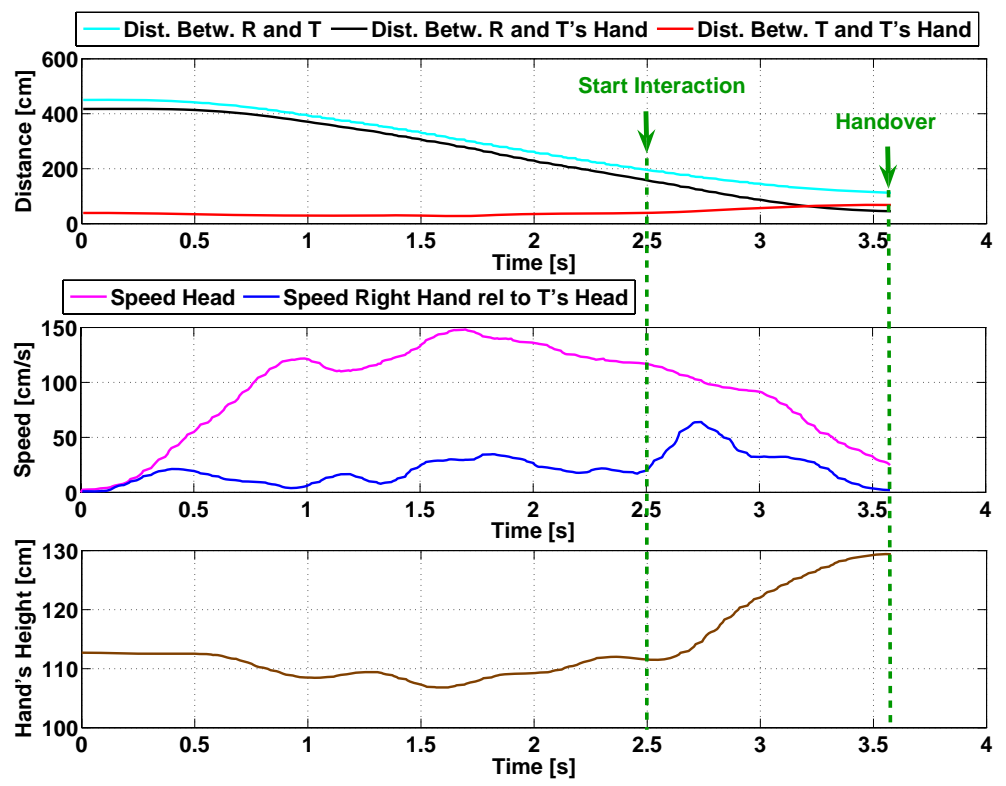

Fig. 2 Definition of the interaction phase.

Furthermore, the distance between $\mathrm{T}$ and his hand at the handover, the distance between $\mathrm{R}$ and T's hand at the handover, the interpersonal distance between $\mathrm{T}$ and $\mathrm{R}$ at the handover, and T's head and hand speed at the handover were determined. The same parameters were derived for the second scenario in which $\mathrm{R}$ had been replaced by a table. For statistical analysis, we used F-tests to compare variability, paired ttests to compare means, and unpaired t-tests to compare against zero. A significance level of 0.05 was adopted in all tests.

\section{Results}

Figure 3 shows two representative examples of head and hand trajectories in top view. Since both subjects were facing each other at the start of each trial, the trajectories recorded for the approaching subject $T$ are close to straight lines [7]. The sinusoidal deviations of the head are caused by the human locomotor pattern, which leads to lateral head deviation with each step.

The results depicted in figures 4 and 5 show that the hand-over position is located closely to the midpoint between $\mathrm{T}$ and $\mathrm{R}$, as found previously for hand-over between 

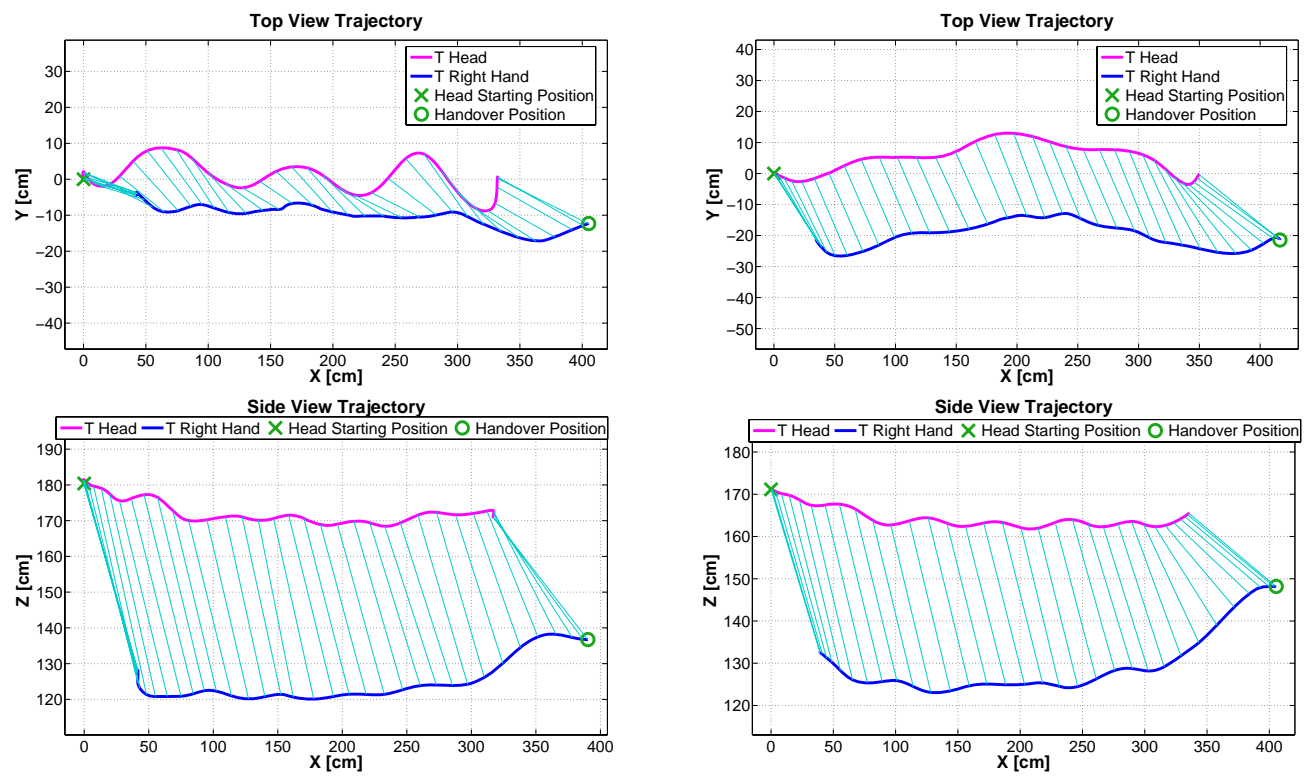

Fig. 3 Two examples of R's head (magenta) and hand (blue) trajectory for the approach and handover. Movement direction is from left to right. Corresponding points in time are connected by lines (cyan).

sitting subjects [8], although shifted slightly but significantly (t-test, $\mathrm{p}<0.05$ ) to the right side (all the probands were right-handed). When expressed in interactionrelated coordinates (right sides of figures 4 and 5), a significant (F-test, $\mathrm{p}<0.05$ ) decrease of the variability of the $\mathrm{X}$ coordinate of the hand-over position was observed (see also table 1).

Table 1 Average and standard deviation of the hand-over position in a global and in an interactionrelated coordinate system

\begin{tabular}{lll}
\hline & Global coordinates $[\mathrm{cm}]$ & Interaction-related coordinates $[\mathrm{cm}]$ \\
\hline Mean & $\mathrm{X}=179.38 \mathrm{Y}=3.6 \mathrm{Z}=140.78$ & $\mathrm{X}=3.88 \mathrm{Y}=3.23 \mathrm{Z}=-35.77$ \\
Standard Deviation & $\mathrm{X}=13.14^{*} \mathrm{Y}=5.33 \mathrm{Z}=5.37$ & $\mathrm{X}=8.09^{*} \mathrm{Y}=3.82 \mathrm{Z}=6.22$ \\
\hline
\end{tabular}

* Variance significantly different (F-test)

Figure 6 shows examples of velocity profiles together with the corresponding height over ground for head and hand. The sinusoidal motion reflects the human locomotor pattern. Head velocity follows a bell-shaped curve: the experiment starts when $\mathrm{T}$ is still standing, then the speed increases while $\mathrm{T}$ approaches $\mathrm{R}$, and finally the speed decreases until the handover. However, even at the actual handover (coinciding with the stop of the measurement, see 2.1), T's head and hand are still moving with a moderate speed. That means, the approaching person stops walking after the 

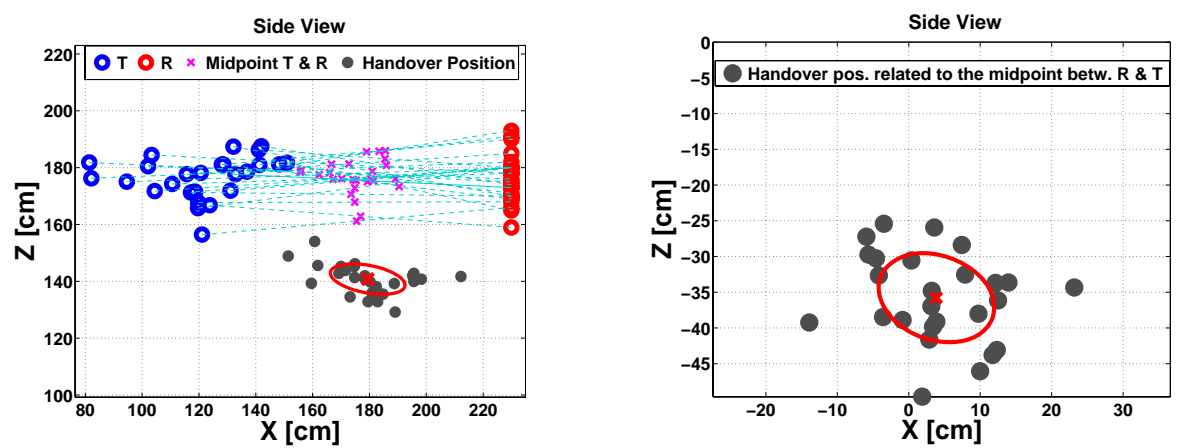

Fig. 4 Left: side view (XZ) of handover positions; the blue circles represent the average position of the head markers of the 26 transferring (T) subjects, the red circles those of the receiving (R) subjects. The purple crosses indicate the mid points between $\mathrm{T}$ and $\mathrm{R}$, and the grey dots the handover positions. Right: handover positions redrawn relative to the midpoint between $\mathrm{R}$ and $\mathrm{T}$ as new origin.
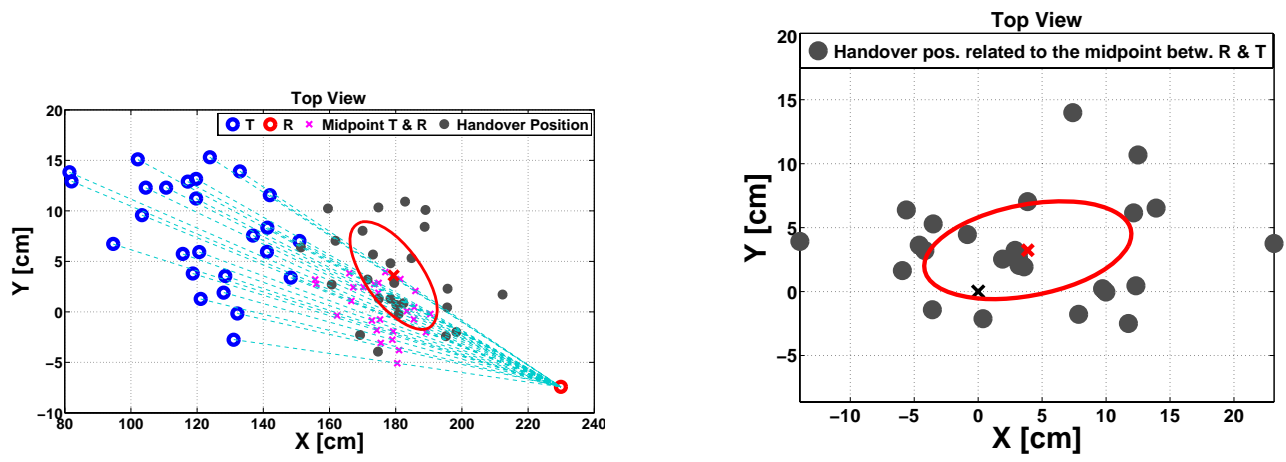

Fig. 5 Top view $(\mathrm{XY})$ of handover positions, same data and same presentation as in figure 4. As seen in the right plot, the lateral position of the handover deviates slightly but significantly to the right side of the receiving subject. Note different axis scaling.

standing person has taken over the object. At the handover, T's head and T's hand had an average speed of $30 \mathrm{~cm} / \mathrm{s}$ and $12.5 \mathrm{~cm} / \mathrm{s}$, respectively.

The results of the second scenario, in which the receiving subject $\mathrm{R}$ was replaced by a table, revealed very similar results. Table 2 compares the average values $( \pm \mathrm{SD})$ of the most relevant parameters for the human-human interaction scenario (left column and figure 7) and the table scenario (right column). For the comparison, data were expressed relative to the handover position for experiment 1 and relative to the table position in experiment 2. A significantly different result was only found for the average peak hand velocity, which was faster in the interaction scenario.

The similarity of both experiments became even more evident when considering not only the average values, but comparing subject by subject. Figure 8 shows that the distance between $\mathrm{T}$ and the object at handover correlates significantly with the distance between $\mathrm{T}$ and the table at the moment of placing the object. Similarly, the 

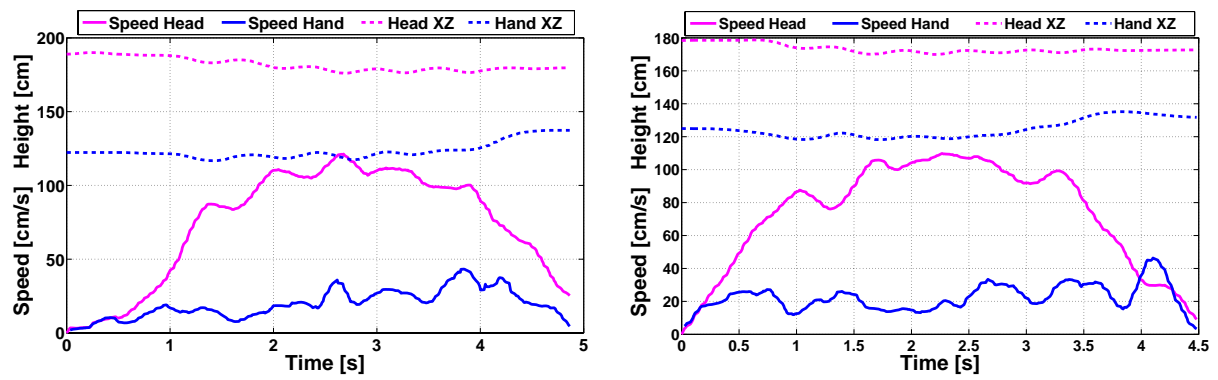

Fig. 6 Examples of velocity profiles and corresponding z-position (height) of head and hand. Hand velocity is expressed with respect to the head.

Table 2 Average parameters $( \pm \mathrm{SD})$ from the two experiments. Left: Interaction scenario; Right: Table scenario

\begin{tabular}{lll}
\hline Parameter & Interaction & Table \\
\hline$d_{1}:$ Distance of T-R at start of handover [cm] & $216,768 \pm 41.95$ & \\
$d_{2}:$ Distance between T at handover initiation and handover & $164.61 \pm 34.19$ & $157.13 \pm 25.97$ \\
position [cm] & & \\
$d_{3}:$ Interpersonal distance at handover [cm] & $116.02 \pm 19.15$ & \\
$d_{4}:$ Distance between T and object at handover [cm] & $64.68 \pm 8.73$ & $69.19 \pm 13.27$ \\
Time between initiation and handover [s] & $1.24 \pm 0.28$ & $1.27 \pm 0.28$ \\
Peak head velocity of T [cm/s] & $133.71 \pm 13.55$ & $128.91 \pm 8.96$ \\
Peak hand velocity of T (relative to head) $[\mathrm{cm} / \mathrm{s}]$ & $114.71 \pm 35.95^{*}$ & $84.8 \pm 22.56^{*}$ \\
\hline
\end{tabular}

* significantly different values for both scenarios $(\mathrm{p}<0.05)$.

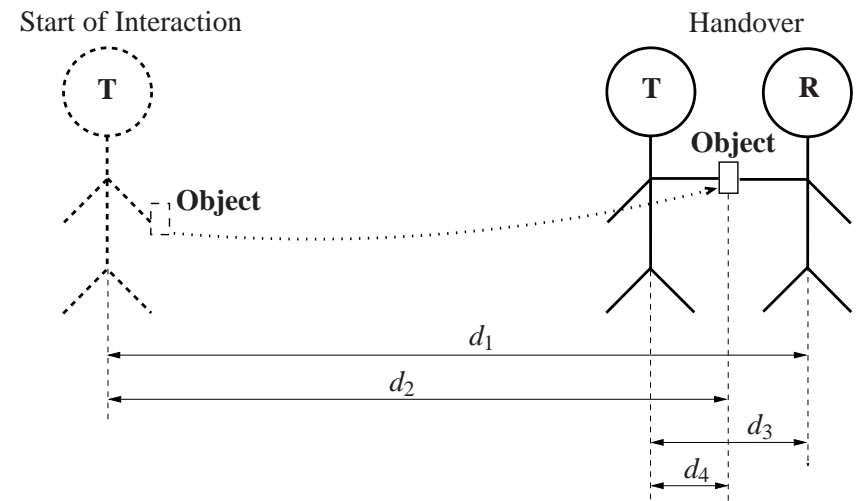

Fig. 7 Approach and handover scenario. The depicted distances $d_{1}, \cdots, d_{4}$ are described in table 2 for the human-human interaction scenario (left column).

distance at which the action is initiated, that is, the lifting of the hand for handover or placing starts, is correlated significantly in both scenarios. 

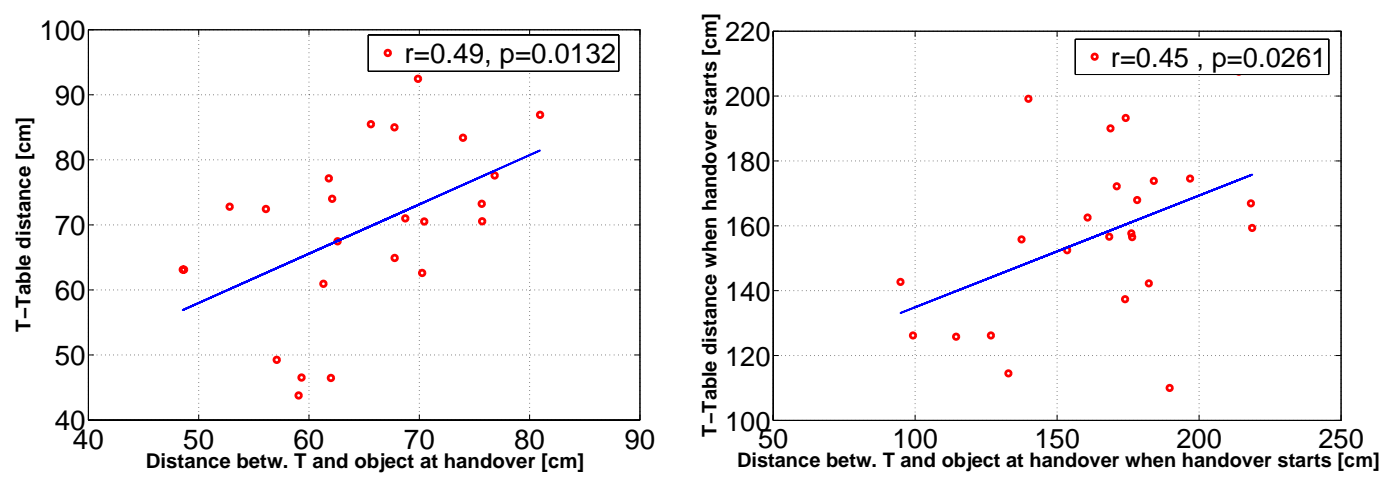

Fig. 8 Correlations between comparable parameters (see also table 2) in both scenarios. Dots represent values from one subject. $\mathrm{X}$-axes: data for handover interaction from $\mathrm{T}$ to $\mathrm{R}, \mathrm{y}$-axis: data for placing of an object on a table by $\mathrm{T}$

We further analysed whether the final distance between both subject depended on the height and/or armlength of $\mathrm{T}$ and/or R. However, no significant correlations between the interpersonal distance at the handover and the height or armlength of the subjects were found.

\subsection{Discussion}

The present results show that approach and handover are smooth and dynamic actions with the different parts blending into each other. For example, the handover is initiated by lifting the object well in advance of the actual object transfer. The transfer itself happens while the transferring person is still moving, and, therefore, the approaching person $\mathrm{T}$ stops walking after the standing person $\mathrm{R}$ has grasped the object. The object transfer occurs at the midpoint between both subjects, thus confirming our earlier results for handover between two sitting subjects [8].

Our second experiment, in which the receiving person was replaced by a table, showed that not only the approach phase for handover or placing the object were very similar, but that even parameters such as distance between head and hand during handover are correlated with the respective parameter while placing the object on a table. That suggests that the large inter-individual differences for certain parameters did not depend on the second, receiving person being present, but were inter-individual preferences, which only depend on the transferring person. While previous work by other groups, e.g. [11], used results from the work of Hall [6] on social interaction to determine factors such as distance between subjects during interaction, our results suggest that, at least in a socially homogeneous group of subjects such as the one used here, additional social factors play a negligible role and the inter-individual variability in hand-over distance represent individual pref- 
erences for action in general.

However, even though the motion of the transferring subject is astonishingly independent of a receiving subject being present, the smooth progress of the handover from early lifting to actual transfer makes it easy for the receiving subject to infer the intention of the transferring partner. Moreover, the final position of the handover is determined by the end position of the transferring subject, and can, due to the smooth velocity trajectory during deceleration, be well predicted by the receiver. Even though we have so far only tested frontal approach, other work [7] has convincingly shown that goal-directed locomotor trajectories follow a maximal-smoothness constraint. Thus, as in the handover itself, where smooth hand trajectories are of importance for intention recognition [9], a smooth locomotor trajectory may be critical for seamless approach and hand-over.

Finally, we would like to point out that several of the present results are directly transferable to the robot. For instance, one can implement in the robot the distance to the receiver when the interaction starts and in addition the handover position. Yet the most important point is possibly that the approach, the start of the interaction by lifting the object appropriately, and the final handover are all performed smoothly and, in part, in parallel instead of being separate and successive motor programs.

Acknowledgements This work is supported by the DFG cluster of excellence "CoTeSys" (www.cotesys.org). Furthermore, we would like to thank our research partner M.Sc. Omiros Kourakos from the Institute of Automatic Control Engineering, Technische Universität München for his support.

\section{References}

1. Alami R, Clodic A, Montreuil V, Sisbot E A, Chatila R (2006) Toward Human-Aware Robot Task Planning. Proceedings of AAAI Spring Symposium: To boldly go where no humanrobot team has gone before, Stanford, USA

2. Althoff D, Kourakos O, Lawitzky M, Mörtl A, Rambow M, Rohrmller F, Brščić D, Wollherr D, Hirche S and Buss M (2009) A flexible architecture for real-time control in multi-robot systems. Human Centered Robotic Systems workshop, this volume

3. Balasuriya J C, Watanabe K, Pallegedara A (2007) ANFIS based active personal space for autonomous robots in ubiquitous environments. International Conference on Industrial and Information Systems:523-528

4. Balasuriya J C, Watanabe K, Pallegedara A (2007) Giving robots some feelings towards interaction with humans in ubiquitous environment. International Conference on Industrial and Information Systems:529-534

5. Fong T, Nourbakhsh I, Dautenhahn K (2003) A survey of socially interactive robots. Robotics and Autonomous Systems:143-166

6. Hall E T (1966) The Hidden Dimension. Doubleday, New York

7. Hicheur H, Pham Q-C, Arechavaleta G, Laumond J-P, Berthoz A (2007) The formation of trajectories during goal-oriented locomotion in humans. I. A stereotyped behaviour. European Journal of Neuroscience 26:2376-2390

8. Huber M, Knoll A, Brandt T, Glasauer S (2009) Handing-over a cube: spatial features of physical joint action. Annals of the New York Academy of Sciences 1164:380-382 
9. Huber M, Rickert M, Knoll A, Brandt T, Glasauer S (2008) Human-robot interaction in handing-over tasks. 7th IEEE International Symposium on Robot and Human Interactive Communication:107-112

10. Kajikawa S, Ishikawa E (2000) Trajectory planning for hand-over between human and robot. 9th IEEE International Workshop on Robot and Human Interactive Communication:281-287

11. Koay K L, Sisbot E A, Syrdal D S, Walters M L, Dautenhahn K, Alami R (2007) Exploratory Study of a Robot Approaching a Person in the Context of Handing Over an Object. Proceedings of AAAI Spring Symposium: Multidisciplinary Collaboration for Socially Assistive Robotics, AAAI Technical Report:18-24

12. Nakauchi Y, Simmons R (2002) A social robot that stands in line. Autonomous Robots 12 (3):313-324

13. Satake S, Kanda T, Glas D F, Imai M, Ishiguro H, Hagita N (2009) How to approach humans? Strategies for social robots to initiate interaction. ACM/IEEE International Conference on Human-Robot Interaction:109-116

14. Sisbot E A, Clodic A, Alami R, Ransan M (2008) Supervision and motion planning for a mobile manipulator interacting with humans. ACM/IEEE International Conference on HumanRobot Interaction:327-334

15. Walters M L, Dautenhahn K, Woods S, Koay K L (2007) Robotic etiquette: results from user studies involving a fetch and carry task. ACM/IEEE International Conference on HumanRobot Interaction:317-324 